\title{
A New Refinement Procedure for Graph Isomorphism Algorithms
}

\author{
Mateus de Oliveira Oliveira ${ }^{1,2}$ \\ Computer Science Department \\ Federal University of Bahia \\ Salvador de Bahia, Brazil \\ Fabíola Greve ${ }^{3}$ \\ Computer Science Department \\ Federal University of Bahia \\ Salvador de Bahia, Brazil
}

\begin{abstract}
Two vertices of a graph are said to be similar if there exists a graph automorphism mapping one of them into the other. Procedures aiming to separate vertices of a graph into equivalence classes accordingly to their similarities are the basis of many isomorphism-testing algorithms. In practice, these procedures efficiently reduce the search space of backtracking-based techniques. We present a new refinement procedure that associates to each pair of vertices the value of an arbitrary predefined invariant. We also propose such a new invariant that we call the least colored shortest path.
\end{abstract}

Keywords: graph isomorphism, refinement procedure, vertex classification

1 This work is supported by CNPQ-Brazil, grant numbers 40.80.86/03-2 and 50.13.89/03-2.

2 Email: mateus@im.ufba.br

3 Email: fabiola@im.ufba.br 


\section{Introduction}

Some algorithms for graph isomorphism testing work in a very acceptable amount of time for most practical purposes. They require an exponential computation time only for a small fraction of graphs. The basis of such practical satisfactory algorithms are procedures that try to color (separate into equivalence classes) the vertices of the graph so that two of them receive the same color if and only if they are similar. In other words, they try to find the orbits of the automorphism group of the graph. No such algorithm is known that does it in polynomial time. Indeed, the problem of determining the orbits of the automorphism group of a graph is both necessary and sufficient for isomorphism testing [3]. In practice, refinement procedures are used to efficiently reduce the search space of backtracking-based techniques. The greater the ability of a procedure to place pairs of non-isomorphic vertices in different classes, the smaller the expected depth of the search tree.

A simple procedure which has been the basis of many practical implementations of the graph isomorphism testing is the one-dimensional WeisfeilerLehman method [1]. This method considers for a fixed vertex only the immediate neighbors in each color class. Miyazaki [5] extends it by taking into account non-immediate neighbors as well, gaining thus in distinction power. In this work we present a new refinement procedure inspired in Miyazaki's extension. Furthermore, we propose a new invariant that relates pair of vertices.

The rest of this paper is organized in the following way. Section 2 describes the 1-dim Weisfeiler-Lehman method. Section 3 presents Miyazaki's extension. This is modified in Section 4 in order to avoid its inherently recursive definition. Our modification also makes it possible to use arbitrary invariants, relating pair of vertices, to increase its distinction power. Section 5 defines such a invariant that we call the least colored shortest path invariant. Associating it to our extended procedure, we obtain a complete refinement algorithm. Finally, in Section 6, we relate our results with previous works and discuss some future directions.

\section{One Dimensional Weisfeiler-Lehman Method}

The one-dimensional Weisfeiler-Lehman method [1], also known as the naive vertex-classification algorithm, is an old idea described in Read and Corneil [6]. It has been used as the basis of implementations of graph isomorphism testing like Mckay's remarkable NAUTY package [4]. The method use information about neighborhoods of vertices of a graph to successively increase the number 
of its color classes repeating the following two steps:

(i) Associate to each vertex $v \in V$ a tuple in which the first position corresponds to its color and the $k$ subsequent ones to the number of its immediate neighbors in each one of the $k$ already existing color classes. Formally, if $X=(V, E)$ is a graph and $\varphi: V \rightarrow \Omega$ is a coloring of $V$, call $d_{i}(v)$ the number of neighbors of $v$ in the $i-t h$ color class and form the tuple

$$
D(v)=\left(\varphi(v), d_{1}(v), d_{2}(v), \ldots, d_{k}(v)\right)
$$

(ii) Define the new color $\varphi^{\prime}(v)$ of $v$ as the ordering number of $D(v)$ in the lexicographical ordering of the set $\{D(v): v \in V\}$.

Let $\varphi^{(j)}$ be the coloring obtained in the $j^{\text {th }}$ iteration of the above procedure. We stop the algorithm when $\varphi^{(r)}=\varphi^{(r+1)}$ to some $r<|V|$. Define $\bar{\varphi}=\varphi^{(r)}$ and call it the stable refinement of $\varphi$.

\section{Miyazaki's Extension}

In [5] instead of counting the number $d_{i}(v)$ of immediate neighbors of the vertex $v$ in the color class of number $i$, Miyazaki extends the naive classification algorithm by defining $d_{i}^{\delta}$ as being the number of nodes in the $i^{\text {th }}$ color class whose distance to $v$ is $\delta$. The tuples $D(v)(v \in V)$ of the original algorithm are substituted by

$$
D_{\delta}(v)=\left(\varphi(v), d_{1}^{\delta}(v), d_{2}^{\delta}(v), \ldots, d_{k}^{\delta}(v)\right)
$$

The new color $\varphi^{\prime}(v)$ of $v$ is defined to be the lexicographic ordering number of $D_{\delta}(v)$. Now we call $S_{\delta}(\varphi)=\varphi^{(r)}=\varphi^{(r+1)}$ the $\delta$-stable refinement of $\varphi$. Note that $\bar{\varphi}$ defined in the naive classification algorithm is just the 1-stable refinement of $\varphi$, namely $S_{1}(\varphi)$.

Miyazaki defines the strong $\delta$-stable refinement of $\varphi$ recursively:

$$
\bar{S}_{\delta}(\varphi)=\left\{\begin{array}{cc}
S_{\delta}(\varphi) & \text { if } \delta=1 \\
S_{\delta}\left(\bar{S}_{\delta-1}(\varphi)\right) & \text { if } \delta>1
\end{array}\right.
$$

\section{Extending Miyazaki's Refinement Procedure}

Now, let us extend the refinement procedure defined by Miyazaki in order to adapt it to use any invariant relating two vertices of the graph and to eliminate recursion in the computation of stable colorings. 
Let $X=(V, E)$ be a graph and consider a function that associates to each pair $u$ and $v$ in $V$, not necessarily an edge, an isomorphism-invariant value $\beta(u, v)$, in a partially ordered set. We redefine $d_{i}^{\delta}(v)$ to be the number of vertices $u$ in the $i^{\text {th }}$ color class of $X$ with $\beta(u, v)=\delta$. We also redefine $D(v)$ as:

$$
D(v)=\left(\varphi(v), \operatorname{SORT}\left\{\left(i, \delta, d_{i}^{\delta}\right): d_{i}^{\delta}>0\right\}\right)
$$

Here SORT is a function that receives as input a finite totally ordered set $T$ and returns the sequence $\left(t_{0}, t_{1}, \ldots, t_{|T|}\right)$ such that $t_{i} \leq t_{i+1}$ for all $0 \leq i<|T|$.

The last part of this refinement procedure is just like the one given in the previous section. Sort $\{D(v): v \in V\}$ lexicographically, and define the new color $\varphi^{\prime}(v)$ of $v$ to be the ordering number of $D(v)$. We call $\bar{\varphi}_{\beta}=\varphi^{(r)}=\varphi^{(r+1)}$ the $\beta$-stable refinement of $\varphi$. Here we can establish our first result. It states that when the invariant $\beta$ associated to our procedure is the distance between vertices, we indeed have an extension of Miyazaki's one.

Lemma 4.1 Let $\beta(u, v)$ be the distance between $u$ and $v$. The $\beta$-stable refinement of $\varphi$ is at least as fine as Miyazaki's strong $\delta$-stable refinement of $\varphi$.

Proof. It suffices to show that a $\beta$-stable refinement of $\varphi$ gives a strong $\delta$ stable refinement for any $\delta$. So, suppose that $\varphi$ is $\beta$-stable. Then, for any pair $u$ and $v$ of vertices belonging to the same color class, $D(u)=D(v)$, i.e., vertices $u$ and $v$ cannot be distinguished. So, the set $\left\{\left(i, \delta, d_{i}^{\delta}(u)\right)\right\}$ in $D(u)$ must be the same as the set $\left\{\left(i, \delta, d_{i}^{\delta}(v)\right)\right\}$ in $D(v)$. But it implies that for any $i$ and $\delta$ the number of elements in the $i^{\text {th }}$ color class that are at distance $\delta$ from $u$, that is to say, $d_{i}^{\delta}(u)$ in Miyazaki's extension is equal to the number of elements in the $i^{\text {th }}$ color class that are at distance $\delta$ from $v$, namely $d_{i}^{\delta}(v)$. Thus $D_{\delta}(u)=D_{\delta}(v)$ for any $u$ and $v$ in the same color class, which makes $\varphi$ also $\delta$-stable for any $\delta$. Finally, this last equality makes $\varphi$ strong $\delta$-stable given the inductive definition of $\bar{S}_{\delta}$.

\section{The Least Colored Shortest Path Invariant}

Now, we define a new invariant relating pairs of vertices of a graph. With the language developed above, just set $\beta$ to be the invariant in our new refinement procedure.

Let $P=x_{1} x_{2} \ldots x_{k}$ be a path joining $x_{1}$ to $x_{k}$. We say that the color of $P$ is the sequence $c(P)$ of colors of the vertices in $P$. That is

$$
c(P)=\left(\varphi\left(x_{1}\right), \varphi\left(x_{2}\right), \ldots, \varphi\left(x_{k}\right)\right)
$$


Let $P$ and $Q$ be two paths from $u$ to $v$ of the same length. We say that $P$ has a smaller color than $Q$ if $c(P)<c(Q)$, lexicographically compared.

Let $\Pi(u, v)$ be the set of all shortest paths from $u$ to $v$. We define $C(u, v)$ to be:

Definition 5.1 [The Least Colored Shortest Path Invariant]

$$
C(u, v)=\min \{c(P): P \in \Pi(u, v) \cup \Pi(v, u)\}
$$

\section{The Least Shortest Path Refinement Procedure:}

Let $X=(V, E)$ be a graph and $\varphi: V \rightarrow \Omega$ be a coloring of $\mathrm{X}$.

(i) Set $\beta=C(u, v)$.

(ii) Find the $\beta$-stable refinement of $\varphi$.

The correctness of the algorithm is implied by our second result stated in the following Lemma.

Lemma 5.2 (i) $C(u, v)$ can be obtained in polynomial time.

(ii) $C(u, v)$ is invariant under isomorphism of colored graphs.

(iii) If $d$ is the distance between vertices then the $C$-stable refinement of $\varphi$ is at least as finer as the $d$-stable refinement of $\varphi$.

\section{Proof.}

Let us sketch a brief proof of item (1), the others can be directly demonstrated from what we have discussed.

The union of all paths in $\Pi(u, v)$ is a DAG that can be easily obtained by an adaptation of the breadth-first search method. In this graph, $u$ is the source and $v$ is the sink. Beginning from $u, L_{i}$ is the $i^{t h}$ level of the DAG. Let $Q$ be any set of vertices. We define $c_{\min }(Q)$ to be the least vertex color in $Q$. Call $\pi$ the size of the paths in $\Pi(u, v)$. Form the sequence $Q_{i}, 1 \leq i<\pi$ in the following way:

(i) $Q_{1}=L_{1}=\{u\}$

(ii) $Q_{i+1}=\left\{p \in L_{i+1}: p\right.$ is reachable from $\left.q \in Q_{i}, \varphi(q)=c_{\min }\left(Q_{i}\right)\right\}$

Then $C(u, v)=\left(c_{\min }\left(Q_{1}\right), c_{\min }\left(Q_{2}\right), \ldots, c_{\min }\left(Q_{\pi}\right)\right)$. It is clear that $C(u, v)$ can be obtained in polynomial time. 


\section{Some Final Remarks}

A generalization of the one-dimensional Weisfeiler-Lehman, namely the $k$ $\operatorname{dim} W$ - $L$ method, classifies not vertices but tuples $\left(u_{1}, u_{2}, \ldots, u_{k}\right)$ of them. An interesting question is whether there exists a low-growth $k$ (or even a constant $k$ ) for which graph isomorphism testing can be performed by the $k$-dim $W$ - $L$ method. This question was negatively answered by Cai, Fürer and Immerman [1]. Using Fürer Gadgets [2], they constructed a class of nonisomorphic pairs of graphs, forcing $k$ to be $\Omega(n)$.

Associated with the 1-dim WL method, the remarkable Mckay's NAUTY algorithm [4] uses information gathered from partial automorphisms and some graph invariants to determine the canonical labeling of graphs. Based on the counterexample given in [1], Miyazaki [5] succeeded in constructing a family of graphs that forces NAUTY to generate exponential number of labelings during the search. This remains true even when the 1-dim WL method is replaced by his extension described in Section 3. In fact, for the elements of this family, independently of which refinement procedure and invariant being used, there will always exists a particular coloring for which NAUTY looses its ability to split color classes of size two.

Instead of treating the isomorphism testing by the point of view of canonical labeling, we prefer the approach of trying to distinguish two graphs or determine an isomorphism between them by direct comparison. The new refinement procedure presented here can be used to improve the distinction effect of any kind of invariant relating pair of vertices. In Lemma 4.1, we prove that the distance between vertices can be used in our procedure to produce at least as finner stable colorings as Myazaki's. Using the distance in conjunction with other invariants (such as the size of the smallest cycle involving two vertices), we can identify classes of graphs for which our alternative in fact produce better refinements.

Instead of considering just the distance between vertices, the least colored shortest path invariant takes into account the information concerning entirely paths. Thus, not only the length but also the way in which the colors in the least colored shortest path alternate could contribute to the distinction of vertices. Currently, we are investigating the existence of pair of graphs that can be distinguished with the help of the least colored shortest path but for which the traditional distance approach does not work.

Finally, we would like to consider a convenient way to generalize our refinement procedure in order to allow the classification of $k$-tuples of vertices instead of single ones. 


\section{References}

[1] J. Cai, M. Fürer and N. Immerman, An Optimal Lower Bound on the Number of Variables for Graph Identification, Combinatorica, 12 (1992), 4, 389-410.

[2] M. Fürer, A Counterexample in Graph Isomorphism Testing, Tech. Rep. CS87-36, Depart. of Computer Science, Pennsylvania State University, 1987.

[3] R. Mathon, A Note on The Graph Isomorphism Counting Problem. Information Proceeding Letters, 8 (1979), 131-132.

[4] B. D. Mckay, Practical Graph Isomorphism, Congressus Numerantium, 30 (1981), 45-87.

[5] T. Miyazaki, The Complexity of Mckay's Canonical Labeling Algorithm, Proc. Groups Computat. II , Work. Groups Computat., DIMACS Series on Discrete Math. Theor. Comput. Sci., L. Finkelstein and W. M. Kantor, eds., 1996.

[6] R. C. Read and D.G Corneil, The Graph Ismorphism Disease, Journal of Graph Theory, 1 (1977), 339-363. 